\title{
HEAT CONDUCTION FOR RIEMANNIAN FOLIATIONS
}

\author{
SEIKI NISHIKAWA, MOHAN RAMACHANDRAN AND PHILIPPE TONDEUR
}

A foliation $\mathscr{F}$ on a manifold $M$ is a partition of $M$ into submanifolds, the leaves of $M$, which locally looks like a family of parallel subspaces in Euclidean space. This gives rise to two types of geometries, namely tangential and transversal.

More specifically let $g_{M}$ be a Riemannian metric on $M$. It induces on each leaf a Riemannian metric, and hence a corresponding leafwise Laplacian $\Delta_{0}$. The action of the corresponding semigroup $e^{-t \Delta_{0}}$ on the bigraded de Rham complex $\Omega_{M}$ is studied in [AT], and leads to a tangential or leafwise Hodge decomposition theorem.

A foliation $\mathscr{F}$ is Riemannian [R], if the induced Riemannian metric $g_{Q}$ on the normal bundle $Q=T M \mid L, L$ the tangent bundle of $\mathscr{F}$, is holonomy invariant, i.e. $\theta(X) g_{Q}=0$ for all vector fields $X$ tangent to $\mathscr{F}$. This gives rise to a transversal Riemannian geometry, which can heuristically be thought of as the Riemannian geometry of the (singular) space of leaves $B=M / \mathscr{F}$. The complex $\Omega_{B}(\mathscr{F}) \subset \Omega_{M}$ of forms $\omega$ satisfying $i(X) \omega=0$ (interior product) and $\theta(X) \omega=0$ (Lie derivative) for all $X \in \Gamma L$ is the complex of basic differential forms of $\mathscr{F}$, and heuristically plays the role of the de Rham complex of the leaf space $B$. The transversal Riemannian metric $g_{Q}$ gives rise to a transversal or basic Laplacian $\Delta_{B}: \Omega_{B}(\mathscr{F}) \rightarrow$ $\Omega_{B}(\mathscr{F})$. The main point of this announcement is to construct and study the corresponding semigroup $e^{-t \Delta_{B}}$ acting on $\Omega_{B}(\mathscr{F})$, and to examine its limit behavior for $t \rightarrow \infty$. This yields in particular a new proof of the Hodge decomposition theorem in $\Omega_{B}(\mathscr{F})$.

The Laplacian $\Delta_{B}=d_{B} \delta_{B}+\delta_{B} d_{B}$ is formally constructed from the transversal Riemannian geometry in the normal bundle in the usual fashion. Since the basic differential forms do not constitute all sections of a vector bundle, the usual elliptic theory does not apply directly. A technical device to handle this situation is to extend $\Delta_{B}: \Omega_{B}(\mathscr{F}) \rightarrow \Omega_{B}(\mathscr{F})$ to a genuine elliptic operator $\tilde{\Delta}: \Omega(M) \rightarrow \Omega(M)$. An explicit construction of such an extension was given in [KT]. This involves the assumption that the mean curvature is constant along the leaves. This hypothesis enters in the explicit calculation of the formal adjoint $\delta_{B}$ of $d_{B}$, and hence also of $\Delta_{B}$. It is further used in the construction of the extension $\tilde{\Delta}$. Formally this hypothesis is expressed by dualizing the usual mean curvature vector field to a 1 -form $\kappa$ (vanishing along the leaves of $\mathscr{F}$ ), and requiring it to be an element of $\Omega_{B}^{1}(\mathscr{F})$. The reason for this assumption is that it makes the

Received by the editors April 28, 1988 and, in revised form, April 19, 1989. 57R30.

1980 Mathematics Subject Classification (1985 Revision). Primary 58G11; Secondary

The third author was in part supported by a grant from the National Science Foundation. 
construction of $\tilde{\Delta}$ as explained above possible. It is not known if the results stated below hold in a more general context. However, the mean curvature hypothesis holds for several interesting classes of foliations, including Riemannian submersions (for appropriate metrics), E. Cartan's isoparametric families of surfaces [C], and foliations by the orbits of isometric Lie group actions ( $B$ is then an orbifold). A typical example of the latter kind is the flow defined by a nonsingular Killing field. The mean curvature form is invariant under the flow, hence constant along the orbits.

For an initial $r$-form $\alpha_{0} \in \Omega_{B}^{r}(\mathscr{F}), 0 \leq r \leq q=\operatorname{codim}(\mathscr{F})$, consider then the heat equation

$$
\frac{\partial}{\partial t} \alpha(x, t)=-\Delta_{B} \alpha(x, t), \quad \lim _{t \downarrow 0} \alpha(x, t)=\alpha_{0}(x) .
$$

In the situation described above, the main result is then as follows.

THEOREM. Let $\mathscr{F}$ be transversally oriented Riemannian foliation on a closed oriented manifold $\left(M, g_{M}\right)$. Assume $g_{M}$ to be a bundle-like metric with $\kappa \in \Omega_{B}^{1}(\mathscr{F})$. Then the following holds.

(i) There exists a unique solution $\alpha$ of (1), given in terms of the fundamental solution $e_{B}^{r}(x, y, t)$ of the basic heat operator $\partial / \partial t+\Delta_{B}$ by

$$
\alpha(x, t)=\int_{M} e_{B}^{r}(x, y, t) \wedge * \alpha_{0}(y)
$$

(ii) Denote $\alpha(x, t)=\left[P_{B}(t) \alpha_{0}\right](x)$. Then there exists a uniform limit

$$
\lim _{t \uparrow \infty} P_{B}(t) \alpha_{0}=H_{B} \alpha_{0} \in \Omega_{B}^{r},
$$

and $H_{B} \alpha_{0}$ is $\Delta_{B}$-harmonic.

(iii) The form

$$
G_{B} \alpha_{0}=\int_{0}^{\infty}\left(P_{B}(t) \alpha_{0}-H_{B} \alpha_{0}\right) d t
$$

is well defined, and gives an operator $G_{B}: \Omega_{B}^{r} \rightarrow \Omega_{B}^{r}$ satisfying

$$
\alpha_{0}=\Delta_{B} G_{B} \alpha_{0}+H_{B} \alpha_{0} \text {. }
$$

The finite-dimensionality of the space of basic harmonic $r$-forms $\mathscr{H}_{B}^{r}=$ $\operatorname{ker} \Delta_{B}$ is a consequence of the method of proof. The identity in (iii) implies in usual fashion the orthogonal Hodge decomposition

$$
\Omega_{B}^{r} \cong \operatorname{im} d_{B} \oplus \operatorname{im} \delta_{B} \oplus \mathscr{H}_{B}^{r}
$$

and the isomorphism $H_{B}^{r} \cong \mathscr{H}_{B}^{r}$ (see [EH, and KT] for proofs of this result). For the case of a point foliation, this is the approach to the classical Hodge decomposition pioneered by Milgram and Rosenbloom [MR].

A typical example is a Riemannian foliation $\mathscr{F}$ transverse to the fibers of a flat bundle $\tilde{X} \times_{\Gamma} F$, defined by an isometric action $h: \pi_{1}(X)=\Gamma \rightarrow \operatorname{Iso}(F)$ of $\Gamma$ on the Riemannian manifold $F$. In this case $\Omega_{B}(\mathscr{F}) \cong \Omega(F)^{\Gamma}$, and the heat flow discussed is induced by the $\Gamma$-equivariant heat flow of the Laplacian on $F$.

The novel technical aspect in the present context is the use of the elliptic extension $\tilde{\Delta}$. For the case of basic functions (forms of degree 0 ) 
treated in [EK] this difficulty does not arise, since the ordinary Laplacian on $M$ already preserves basic functions. The intuitive idea in part (i) of the Theorem is to think of the flow of the basic heat operator $\partial / \partial t+\Delta_{B}$ as arising from the flow of the heat operator $\partial / \partial t+\tilde{\Delta}$ by restriction. The crucial property is then that an initial basic $r$-form $\alpha_{0}$ remains basic under this heat flow. This invariance property constitutes a parabolic version of Hadamard's descent method for linear hyperbolic equations [H]. It is natural to expect that this heat equation approach will be useful for the discussion of the transversal index problem for foliations. Another application is given in [RT], where an almost Lie foliation structure is deformed to a Lie foliation structure by the heat flow method.

\section{REFERENCES}

[AT] J. A. Alvarez-López and Ph. Tondeur, Hodge decomposition along the leaves of a Riemannian foliation (to appear).

[C] E .Cartan, Sur des familles remarquables d'hypersurfaces isoparamétriques dans les espaces sphériques, Math. Z. 45 (1939), 335-367.

[EH] A. El Kacimi et G. Hector, Décomposition de Hodge basique pour un feuilletage riemannien, Ann. Inst. Fourier (Grenoble) 36 (1986), 207-227.

[EK] A. El Kacimi, Equation de la chaleur sur les espaces singuliers, C. R. Acad. Sci. Paris t. 303 (1986), 243-246.

[GR] P. Greiner, An asymptotic expansion for the heat equation, Arch. Rational Mech. Anal. 41 (1971), 163-218.

[H] J. Hadamard, Le problème de Cauchy et les équations aux derivées partielles linéaires hyperboliques, Hermann et Cie, Paris, 1932.

[KT] F. W. Kamber and Ph. Tondeur, De Rham-Hodge theory for Riemannian foliations, Math. Ann. 277 (1987), 415-431.

[MR] A. N. Milgram and P. C. Rosenbloom, Harmonic forms and heat conduction I: Closed Riemannian manifolds, Proc. Nat. Acad. Sci. 37 (1951), 180-184.

[NRT] S. Nishikawa, M. Ramachandran and $\mathrm{Ph}$. Tondeur, The heat equation for Riemannian foliations, Trans. Amer. Math. Soc. (to appear).

[R] B. Reinhart, Foliated manifolds with bundle-like metrics, Ann. of Math. (2) 69 (1959), 119-132.

[RT] E. A. Ruh and Ph. Tondeur, Almost Lie foliations and the heat equation method, Proc. of the VI Internat. Colloq. on Differential Geometry, Santiago de Compostela (Spain) 1988, Cursos y Congresos 10, Univ. of Santiago de Compostela, 1989, pp. 239-246.

Department of Mathematics, Faculty of Science, Kyushu University, Fukuoka 812 JAPAN (CURRENT ADDRESS OF SEIKI NishikAWA)

Department of Mathematics, The Ohio State University, 231 West 18th Avenue, Columbus, OHio 43210

Department of Mathematics, University of Illinois, 1409 West Green Street, Urbana, ILlinoIs 61801 (CURRENT ADDRESS OF MOHAN RAMACHANDRAN AND PhILIPPE TONDEUR) 
